\title{
A Multidimensional Multiplexing Mode-Locked Laser Based on a Dual-Ring Integrative Structure for Tri-Comb Generation
}

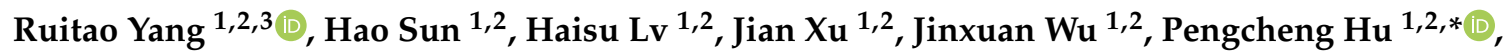 \\ Haijin Fu ${ }^{1,2}$, Hongxing Yang ${ }^{1,2}$ and Jiubin Tan ${ }^{1,2}$ \\ 1 Center of Ultra-Precision Optoelectronic Instrument Engineering, Harbin Institute of Technology, \\ Harbin 150080, China; ruitao.yang@hit.edu.cn (R.Y.); sunhao@hit.edu.cn (H.S.); \\ lvhaisu123@stu.hit.edu.cn (H.L.); 19S101131@stu.hit.edu.cn (J.X.); jinxuan.wu@hit.edu.cn (J.W.); \\ haijinfu@hit.edu.cn (H.F.); yanghx@hit.edu.cn (H.Y.); jbtan@hit.edu.cn (J.T.) \\ 2 Key Lab of Ultra-Precision Intelligent Instrumentation (Harbin Institute of Technology), \\ Ministry of Industry and Information Technology, Harbin 150080, China \\ 3 Postdoctoral Research Station of Optical Engineering Harbin Institute of Technology, Harbin 150080, China \\ * Correspondence: hupc@hit.edu.cn
}

Received: 12 October 2020; Accepted: 19 November 2020; Published: 21 November 2020

Featured Application: This work proposes a dual-ring integrated mode-locked laser to generate a tri-comb for the multi-heterodyne detection technique in the field of laser ranging and precision spectroscopy.

\begin{abstract}
The tri-comb-based multi-heterodyne detection technique has been proven to be a powerful tool for precision metrology, e.g., laser ranging and spectroscopy. However, in existing tri-comb generation methods, it is difficult to provide a large and variable difference in tri-comb repetition rates. In this paper; we propose a multidimensional multiplexing mode-locked laser based on a dual-ring integrative structure. Combining the dimensions of sub-ring multiplexing and wavelength multiplexing, two modes of tri-comb generation can be achieved with the dual-ring single cavity laser. The generated combs are identified based on the relative intensity of the pulse trains and optical spectrum, and the repetition rates of dual-combs from the same sub-ring are distinguished based on dispersion analysis. With repetition rates of approximately $47 \mathrm{MHz}$ and $49.6 \mathrm{MHz}$, the minimum and maximum repetition rate difference of the generated tri-comb can be changed from $2.38 \mathrm{kHz}$ and $2.59526 \mathrm{MHz}$ to $2.74 \mathrm{kHz}$ and $2.59720 \mathrm{MHz}$ merely by switching the operation mode of the dual-ring integrated mode-locked laser. The obtained results indicate that our method can offer a powerful scheme for future multi-comb generation and its application in multi-heterodyne detection-based laser ranging and spectroscopy.
\end{abstract}

Keywords: frequency comb generation; mode-locked laser; tri-comb; multi-heterodyne detection; metrology; spectroscopy; laser ranging

\section{Introduction}

The application of dual-combs with slightly different repetition rates has enabled a revolutionary new technique for the field of detection and metrology, e.g., laser ranging [1-4], velocity measurement [5] and precision spectroscopy [6-8]. By using the dual-comb-based multi-heterodyne detection technique, temporal and spectral information can be extracted with a high resolution and high update-rate simultaneously. Based on the advantages of dual-comb applications, a third comb has been introduced to expand the measurement range in laser ranging [9]. A basic requirement of this tri-comb laser source 
for multi-heterodyne detection is the different scale of differences among the repetition rates of the tri-comb. In the application of large-range, precision and rapid laser ranging, the desired differences of repetition rates should be variable from $\mathrm{kHz}$ to $\mathrm{MHz}$ to enable large range coarse measurements and short-range precision measurements [9]. However, the existing dual-comb generation schemes can hardly fulfill the requirements for the tri-comb technique, as has been discussed in a previous review paper [10]. One conventional dual-comb generation scheme relies on the combination of two independent fully-stabilized or free-running comb sources [1,3-8]. However, the obvious disadvantage of this scheme is the system's complexity and expensiveness. Another approach to dual-comb generation is based on the multiplexing of parameters of a single-cavity laser. The natural good coherence of generated combs and the simple system structure of this method are very attractive even for multi-comb generation. Based on the existing reports, the multiplexing parameters for dual-comb generation include the propagation direction [11-18], polarization [19-21], wavelength [22-25] and pulse shape $[18,26,27]$. Limited by the single-ring laser cavity, however, the application of any single multiplexing can hardly generate more than two combs. Although tri-combs and even quad-combs have been reportedly generated by wavelength multiplexing [18], the instability and difficulty of their realization prohibits a broader application of the scheme.

In 2019, the research group of Prof. Zheng Zheng proposed a method of multi-comb generation using multidimensional multiplexing [28]. By combining the multiplexing of polarization and wavelength, a tri-comb and a quad-comb were generated in high quality. This is an innovative direction for multi-comb generation. The only drawback is that the repetition rates of generated combs are still close to each other. Thus, the multidimensional multiplexing method does not conclusively solve the problems with existing multiplexing schemes. It is still worthwhile to study methods for the generation of multi-combs with large-range and variable repetition rate differences.

Based on our previous studies of the dual-ring mode-locked laser [29], we have found that it is an effective and simple way to achieve variable repetition rates. Similar dual-ring lasers were reported in $[26,27]$ to generate different types of pulses, e.g., dissipative and conventional solitons or vector and scalar pulses. However, the lack of mode-locking evolution analysis has prohibited its application as a multiplexing parameter for simultaneous multi-comb generation. In this paper, a multidimensional multiplexing mode-locked laser based on a dual-ring integrative structure is proposed for tri-comb generation. To our knowledge, this is the first attempt to employ sub-ring multiplexing in multidimensional multiplexing lasers to generate multi-combs. To prevent the requirement of high-cost equipment for polarization-maintaining fiber splicing, the whole laser is composed only of single-mode fibers and free space. Wavelength multiplexing is applied as the other dimension for tri-comb generation. Two modes of tri-comb generation can be realized with the proposed laser. Accordingly, the minimum and maximum difference of repetition rates are $2.38 \mathrm{kHz}$ and $2.59526 \mathrm{MHz}$ in one mode. They can be switched to $2.74 \mathrm{kHz}$ and $2.59720 \mathrm{MHz}$ by just changing the mode of tri-comb generation. We believe the proposed new dimension of sub-ring multiplexing and the dual-ring integrated mode-locked laser could meet the needs of multi-comb generation with repetition rate differences in a large range.

\section{Experimental Setup}

The structure of the multidimensional multiplexing mode-locked laser is shown in Figure 1. Although it is an integrated laser, its dual-ring structure enables extra sub-ring multiplexing for multi-comb generation. This dual-ring structure is realized with a shared common-path and two symmetrical sub-paths. In the common-path, a 0.5-m-long erbium-doped fiber (EDF, Liekki ER110-4/125) is bidirectionally pumped by two 980-nm laser diodes via wavelength-division multiplexers (WDMs). The WDMs in our setup transmit and reflect lights of $1550 \mathrm{~nm}$ and $980 \mathrm{~nm}$, respectively. The common path acts as the gain medium of the whole dual-ring laser. To ensure the unidirectional propagation of the laser in each sub-path, two circulators are applied to connect the common path and two sub-paths. Accordingly, two sub-rings are formed and labelled as R-1 (red) and R-2 (blue), respectively. According 
to the previous studies presented in [21], a passive cancellation of the common mode noise can be achieved with this system structure.

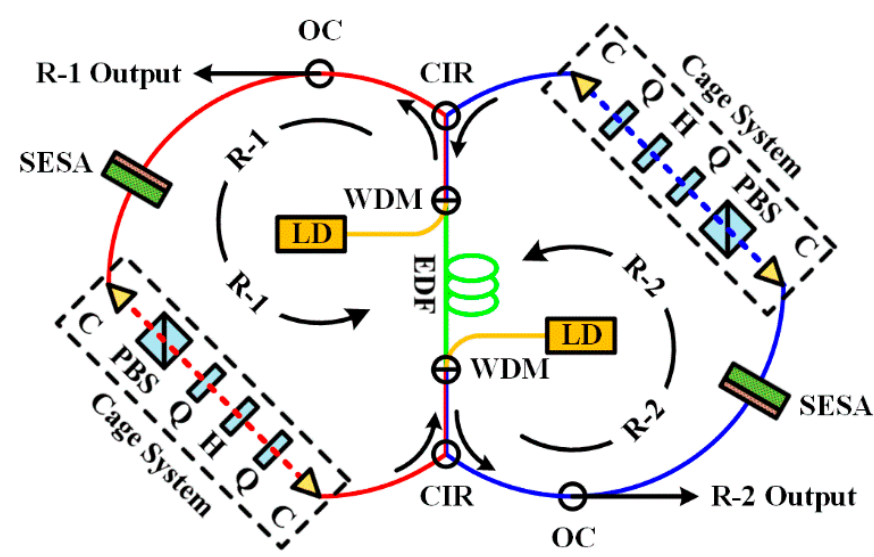

Figure 1. The experimental setup of the multidimensional multiplexing mode-locked laser. EDF, erbium-doped fiber; WDM, wavelength-division multiplexer; CIR, circulator; LD, laser diode; OC, optical coupler; SESA, semiconductor saturable absorber; $\mathrm{C}$, collimator; PBS, polarizing beam splitter; $\mathrm{H}$, half-wave plate; $\mathrm{Q}$, quarter-wave plate; R-1, sub-ring 1 and R-2, sub-ring 2.

In each sub-ring, a semiconductor saturable absorber (SESA, Batop SA-1550-25-2ps) is applied to achieve the self-starting mode-locking. To ensure a free but recoverable switching among the mode-locking states of the dual-ring integrated laser, a cage module is added in each sub-ring to adjust the intracavity loss. The evolution of the mode-locking status of this dual-ring laser has been experimentally explored in our previous works, reported in [29]. Although only dual-comb generation was analyzed, the law of mode-locking evolution for this dual-ring laser is invariable. The simultaneous mode-locking in both sub-rings always requires a balance of power in the dual-ring structure. This can only be achieved by adjusting the intracavity loss of each sub-ring precisely and continuously. Therefore, the existence of these two cage modules is important to some extent for the proposed multidimensional multiplexing mode-locked laser. Although the function of intracavity loss control can be realized by the in-line tunable fiber optical attenuator as well, it is quite hard to record the mode-locking status and to switch among them in the experiments. In comparison, intracavity loss control with the cage module can be realized in two ways. The combination of rotating scaled zero-order wave plates (Thorlabs, WPH05M-1550 and WPQ05M-1550) and a fixed polarizing beam splitter (Thorlabs, PBS104) ensures precise, continuous and periodically repeated loss control. Furthermore, the micrometers for the aiming adjustment of the collimators enable large-range, precise and recoverable loss control. Moreover, the design of such a free space cage module is required for repetition rate stabilization in the near future. The free space length of both the cage modules are adjusted to be approximately $0.2 \mathrm{~m}$.

In contrast to the existing multi-comb generation method using a single-ring mode-locked fiber laser, the new dimension of the sub-ring enables various resonance lengths for the generation of individual combs. Accordingly, the repetition rate of the generated combs can be changed within a large range. The repetition rate can be estimated with the parameters of ring laser as follows.

$$
f_{\text {rep }}=\frac{c}{L_{\mathrm{eqvR}}}=\frac{c}{n_{1} L_{1}+n_{2} L_{2}+\cdots+n_{m} L_{m}},
$$

where $L_{\text {eqvR }}$ stands for the equivalent optical length of the ring laser; $c$ is the speed of light in a vacuum; $n_{1}, n_{2}$ and $n_{\mathrm{m}}$ stand for the refractive index of each segment in the ring laser; and $L_{1}, L_{2}$ and $L_{\mathrm{m}}$ are the length of each segment in the ring laser. In our setup, R-1 (including the common path and red sub-path shown in Figure 1) is composed of 0.5 m EDF fiber, $3.7 \mathrm{~m}$ SMF-28e+ fiber and $0.2 \mathrm{~m}$ free space path. If the refractive index of EDF and SMF-28e+ are both estimated as 1.4679 [30], 
the equivalent optical length of the fibers can be estimated as $6.1652 \mathrm{~m}$. In the $0.2-\mathrm{m}$ free space path, the equivalent optical length of three zero-order wave plates can be neglected. The polarization beam splitter of Thorlabs PBS104 is a 10-mm cube made of N-SF1, of which the refractive index is 1.6832 at $1550 \mathrm{~nm}$ [31,32]. Considering the refractive index of air as 1.00027 [33], the equivalent optical length of the free space path can be estimated as $0.2069 \mathrm{~m}$. With the total equivalent optical length of R-1 being $6.3721 \mathrm{~m}$, the repetition rate of the comb generated from R-1 can be calculated as approximately $47 \mathrm{MHz}$. In comparison, the length of the SMF-28e+ fiber in R-2 is changed to approximately $3.48 \mathrm{~m}$. The total equivalent optical length of R-2 is $6.0442 \mathrm{~m}$. Accordingly, the repetition rate of R-2 should be varied to $49.6 \mathrm{MHz}$ with the same calculation method above. It is clear that the slight difference between repetition rates of the combs from two sub-rings is introduced by the new dimension of the sub-ring structure. More importantly, this new dimension of the sub-ring structure enables a free variation of the difference between repetition rates. This is highly desired in many applications of multi-comb interferometry [2,3,9].

As the group velocity dispersion (GVD) of the EDF and SMF-28e+ are $+0.012 \mathrm{ps}^{2} / \mathrm{m}$ and $-0.022 \mathrm{ps}^{2} / \mathrm{m} @ 1550 \mathrm{~nm}$, respectively [18,23], the net GVD of R-1 and R-2 can be estimated as $-0.0732 \mathrm{ps}^{2}$ and $-0.0710 \mathrm{ps}^{2}$, respectively, when neglecting the dispersion from the short space path. Therefore, both sub-rings in the current setup operate in the anomalous-dispersion regime. Optical couplers are applied to extract $10 \%$ power of the intracavity light as output. The multi-combs generated from the multidimensional multiplexing mode-locked laser were detected by photodetectors with $1.8 \mathrm{GHz}$ bandwidth (Menlo Systems, APD310) and $150 \mathrm{MHz}$ bandwidth (Thorlabs, PDA05CF2). The latter was applied to monitor the repetition rates only. An oscilloscope with a $2.5 \mathrm{GHz}$ sampling rate (Agilent Technologies, MSO9254A) and a spectrum analyzer with $3.6 \mathrm{GHz}$ bandwidth (Agilent Technologies, N9010A) was applied to monitor the pulse train in the time domain and the radio frequency (RF) spectrum of the interference signal in the frequency domain, respectively. The optical spectrum and power of the generated combs were detected by an optical spectrum analyzer (YOKOGAWA, AQ6370C) and a dual-channel optical power meter (Thorlabs, PM320E).

\section{Results and Discussions}

With the proposed dual-ring integrated laser, the generation of a tri-comb requires the generation of a dual-comb from one of the sub-rings. Owning to the single direction propagation property of the circulators, direction multiplexing cannot be achieved in the sub-rings of our setup. Considering that polarization multiplexing cannot be achieved with the proposed all single-mode fiber laser either, the dimension of wavelength multiplexing was added to generate a dual-comb from one sub-ring. By coupling a 400-mW pump laser from each WDM into the EDF, tri-comb generation was stably achieved. The reasons a total pump power as high as $800 \mathrm{~mW}$ was required are as follows. Firstly, the applied EDF fiber was high-concentration doped. The peak core absorption is $110 \mathrm{~dB} / \mathrm{m}$ at $1530 \mathrm{~nm}$. It is reasonable to believe the absorption rate is higher than other EDF fibers at $980 \mathrm{~nm}$ as well. Secondly, the realization of dual-wavelength mode-locking usually requires a higher pump power. It also requires a precise adjustment of cavity loss to change the gain profile of the laser [28], which is achieved by the cage modules in the proposed laser. However, these essential cage modules introduce approximate $40 \%$ power loss for each sub-ring in the fiber-space-fiber coupling process, even under the optimized collimation state and the minimized intracavity loss with wave plates. Moreover, either the collimation or the wave plates should always be adjusted away from the optimized state to maintain the power balance between the dual-rings [29].

According to the discussion above, there are two modes of tri-comb generation with the proposed multidimensional multiplexing mode-locked laser. In the first mode, there are two combs of different wavelengths generated from R-1. At the same time, R-2 outputs only one comb simultaneously. Figure 2 illustrates the pulse train and spectral characteristics of the tri-comb generated through this mode. 

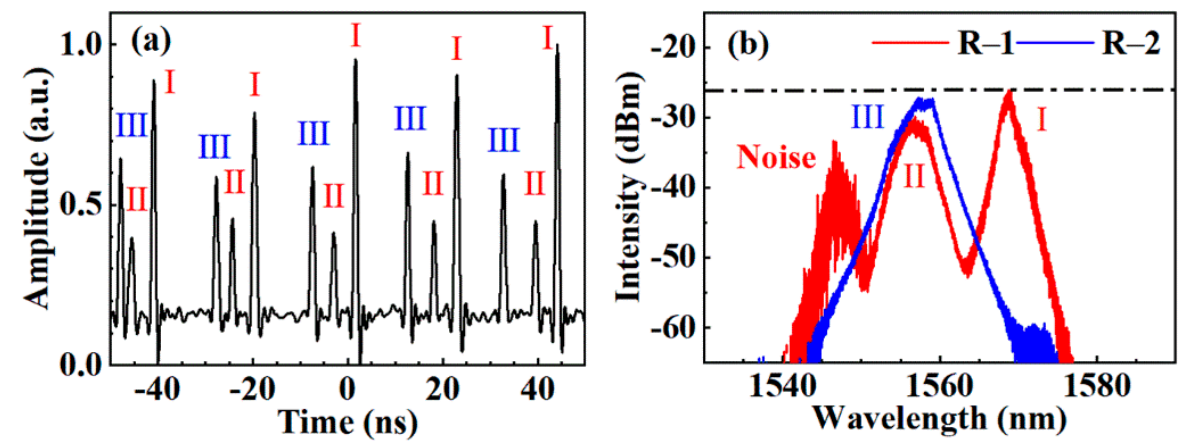

Figure 2. The pulse train and spectral characteristics of the generated tri-comb with dual-comb and single-comb output from sub-ring 1 and sub-ring 2, respectively. (a) Pulse trains, (b) optical spectrum. I and II stand for the pulse trains from sub-ring 1, whereas III stands for the pulse train from sub-ring 2. R-1, sub-ring 1. R-2, sub-ring 2.

In our setup, a $1 \times 2$ fiber coupler with a 50:50 coupling ratio is reverse applied to combine the tri-comb from different output ports of R-1 and R-2 into a single fiber for the following pulse train and optical spectrum detection. In Figure 2a, the pulse trains from the dual-ring laser of both R-1 and R-2 are detected with one photodetector. Three stable trains of pulses can be observed with different time intervals and optical intensities, which are marked with labels of I, II and III, respectively. It is clear that the spacing between pulse trains I and II is relatively constant, compared to the spacing between pulse trains II and III. This phenomenon is caused by the intentionally introduced difference in resonance length for the sub-rings. A more detailed discussion of the repetition rates will be performed in the following paragraphs. According to this effect, we can identify that pulse trains I and II are generated from R-1 and pulse train III is generated from R-2. For the optical intensities, despite the peak amplitudes changing over time, the relative intensity among the three pulse trains is still clear enough to distinguish them. More importantly, this relative intensity can be applied to identify the pulse trains from the peaks of the detected optical spectrum.

In Figure $2 b$, the red solid line and blue solid line stand for the optical spectrum from the separated outputs of R-1 and R-2, respectively. For the output spectrum of R-1, two obvious spectral peaks can be found with different peak intensities. With the help of the relative intensity shown in Figure 2a, the average power of comb I can be estimated to be higher than that of comb II. Thus, the stronger peak of $1569.1 \mathrm{~nm}$ center wavelength and $1.4 \mathrm{~nm}$ bandwidth in Figure $2 \mathrm{~b}$ corresponds to pulse train I. Centered on a $1556.1 \mathrm{~nm}$ wavelength, the weaker peak of $3.3 \mathrm{~nm}$ bandwidth in Figure $2 \mathrm{~b}$ is the optical spectrum of pulse train II, depicted in Figure 2a. A noise peak with an unstable intensity and shape can be observed at approximately $1546.5 \mathrm{~nm}$ in Figure $2 \mathrm{~b}$ as well. We attribute this to the excess energy in R-1. A fine adjustment of the wave plates or the space collimation in cage modules should remove this noise peak. However, the stability of the dual-wavelength comb generation in R-1 was affected in our experiments. We will do more research on this to further improve the results in the near future. For the output spectrum of R-2, depicted by a blue solid line, the only peak is centered on $1557.3 \mathrm{~nm}$ with a bandwidth of $4.0 \mathrm{~nm}$.

Figure 3 illustrates the radio frequency (RF) characteristics of the generated tri-comb with dual-comb and single-comb outputs from R-1 and R-2, respectively. The outputs from R-1 and R-2 were individually photodetected and monitored by use of a spectrum analyzer with a resolution bandwidth (RBW) of $180 \mathrm{~Hz}$. In Figure 3a, two strong peaks of $47.00198 \mathrm{MHz}$ and $47.00436 \mathrm{MHz}$ can be observed from R-1 with a signal-to-noise ratio (SNR) of over $40 \mathrm{~dB}$. In comparison, there is only one peak of 49.59724 MHz from R-2 shown in Figure 3b. Considering the measurement error of each segment fiber, the experimental result of repetition rates from R-1 and R-2 fit very well with the theoretical analysis presented in Section 2. 

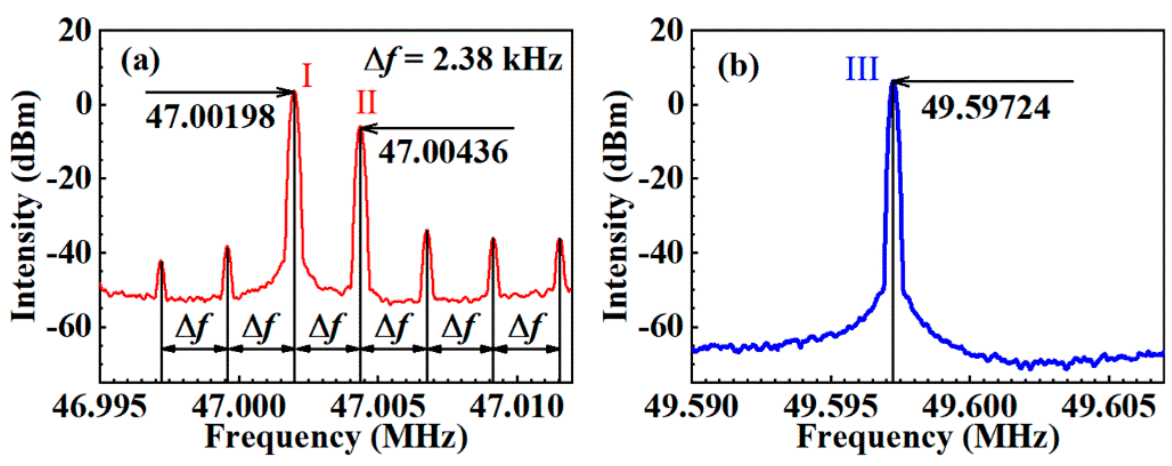

Figure 3. The radio frequency (RF) spectral characteristics of the generated tri-comb with dual-comb and single-comb output from sub-ring 1 and sub-ring 2, respectively. (a) RF spectrum of sub-ring 1 and (b) RF spectrum of sub-ring 2.

In Figure 3a, there is a slight difference of $2.38 \mathrm{kHz}$ between the two repetition rates of comb I and II from the same R-1. We attribute this phenomenon to the dispersion of different center wavelengths. A brief theoretical analysis can be conducted as follows. According to the definition of repetition rate, the ratio of dual-comb repetition rates from $\mathrm{R}-1$ can be calculated as

$$
\frac{f_{\text {rep } \mathrm{I}}}{f_{\text {rep II }}}=\frac{\frac{c}{n_{g I} L}}{\frac{c}{n_{g I I} L}}=\frac{n_{g \mathrm{II}}}{n_{g \mathrm{I}}},
$$

where $f_{\text {repI }}$ and $f_{\text {rep II }}$ stand for the repetition rates of pulse trains I and II, respectively; $L$ stands for the equivalent geometrical length of R-1; and $n_{g I}$ and $n_{g I I}$ represent the equivalent group refractive index for pulse trains I and II, respectively. As the real R-1 is composed of several different types of fibers and some free space, the equivalent geometrical length and equivalent group refractive index are applied here to accomplish the rough analysis. Based on this approximation, an inverse relation between the repetition rate and the equivalent group refractive index is revealed in Equation (2). As discussed in Section 2, both sub-rings operate in the anomalous-dispersion regime, where the group velocity dispersion parameter of $\beta_{2}$ is a negative number. According to the definition of $\beta_{2}$ in [34], the relationship between equivalent group refractive index $n_{g}$ and optical frequency $\omega$ can be expressed as

$$
\beta_{2}=\frac{1}{c} \frac{\mathrm{d} n_{g}}{\mathrm{~d} \omega}<0
$$

According to Equations (2) and (3), an inverse relationship between the repetition rate and center wavelength of generated combs can be derived, considering the inverse relationship between wavelength and optical frequency. Therefore, the left stronger peak of $47.00198 \mathrm{MHz}$ repetition rate in Figure 3a belongs to pulse train I and the right weaker peak of $47.00436 \mathrm{MHz}$ corresponds to pulse train II. This result is consistent with the graphical analysis of relative intensity as well.

It should be noticed that the maximum repetition rate difference of $2.59526 \mathrm{MHz}$ was obtained with comb I and III generated from different sub-rings. Introduced by the intentional length variation in the dimension of sub-ring multiplexing, this significant difference in repetition rates is actually hard to achieved with other multi-comb multiplexing generation schemes, e.g., direction multiplexing, wavelength multiplexing, polarization multiplexing and temporal pulse shaping multiplexing. More importantly, the proposed new dimension of sub-ring multiplexing enables a free and large-range adjustment of the repetition rate difference.

In Figure 3a, it is also very interesting to find some equally spaced small peaks on both sides of the desired signals. We attribute these signals to the extra interference among the RF signals of combs I and II. The basic principle of this phenomenon can be explained with the help of Figure 4 . The self-interference signal of combs I and II can be seen with the RF spectrum in Figure 4a. In addition 
to the desired inter-mode interference of combs I and II, the extra interference of a different order of comb modes leads to the side peaks of spectrum shown in Figure $4 \mathrm{~b}$. As the number of comb modes needed to generate these extra interferences is much less than the modes for the repetition rate, the SNR of these extra peaks is usually less than $20 \mathrm{~dB}$.

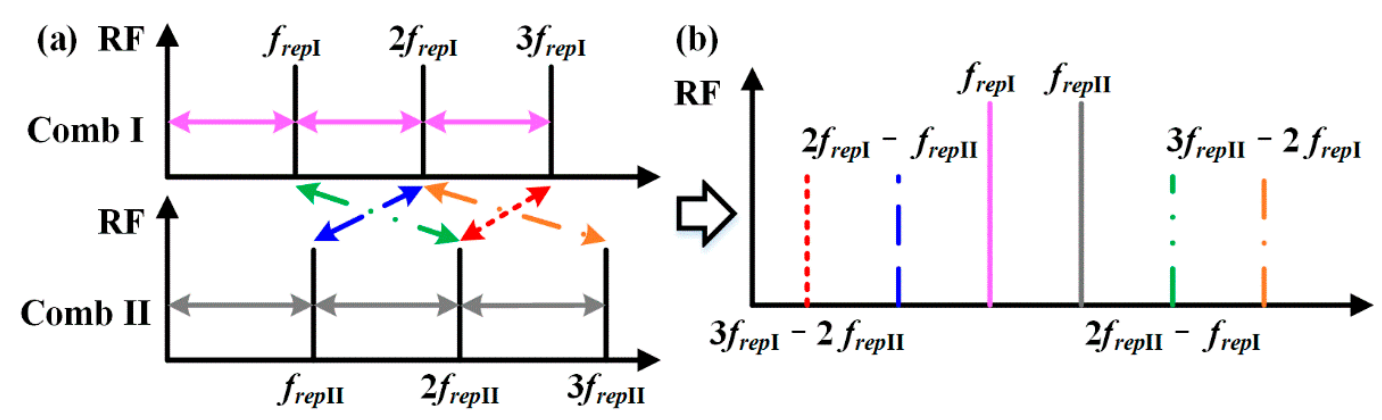

Figure 4. The schematic of RF spectrum generation for dual-combs I and II from sub-ring 1. (a) The individual RF spectrum of combs I and II. (b) The final RF spectrum output from sub-ring 1.

In the other tri-comb generation mode of the proposed multidimensional multiplexing mode-locked laser, only one comb is generated from R-1, while the R-2 outputs a dual-comb of two wavelengths simultaneously. The characteristics of the generated tri-comb in this mode are illustrated in Figure 5. In Figure 5a, the pulse trains are shown, designated as IV, V and VI. From the spacing among the pulse trains, it can be confirmed that only pulse train IV comes from R-1, whereas R-2 outputs the pulse trains of V and VI. Again, the relative intensity of pulse trains corresponds very well with the optical spectrum shown in Figure 5b. Thus, the spectral peaks can be easily identified. In this case, the center wavelength of comb IV is approximately $1564.5 \mathrm{~nm}$ and the center wavelengths of combs V and VI are approximately $1546.1 \mathrm{~nm}$ and $1568.1 \mathrm{~nm}$, respectively. Accordingly, the optical spectrum bandwidth of combs IV, V and VI are $1.9 \mathrm{~nm}, 1.4 \mathrm{~nm}$ and $1.7 \mathrm{~nm}$, respectively. With the same RBW of $180 \mathrm{~Hz}$ as before, the RF spectra of combs IV, V and VI were detected as well. In Figure 5c, the only spectral peak reveals a repetition rate of $47.00200 \mathrm{MHz}$ for comb IV from R-1. In comparison, the repetition rates of combs V and VI in Figure $5 \mathrm{~d}$ can be confirmed as $49.59920 \mathrm{MHz}$ and $49.59646 \mathrm{MHz}$ with the same methods discussed above, respectively. A constant frequency difference of $2.74 \mathrm{kHz}$ can be found between the spectral peaks shown in Figure $5 \mathrm{~d}$. Due to the difference in sub-ring length, the minimum and maximum repetition rate differences are changed from $2.38 \mathrm{kHz}$ and $2.59526 \mathrm{MHz}$ to $2.74 \mathrm{kHz}$ and $2.59720 \mathrm{MHz}$ merely by switching the mode of tri-comb generation. This further proves the variability of the proposed multidimensional multiplexing mode-locked laser. The continuous mode-locking of both the tri-comb generation modes can last for over $10 \mathrm{~h}$. In future studies, we will develop a stabilization system for the proposed dual-ring mode-locked laser. 

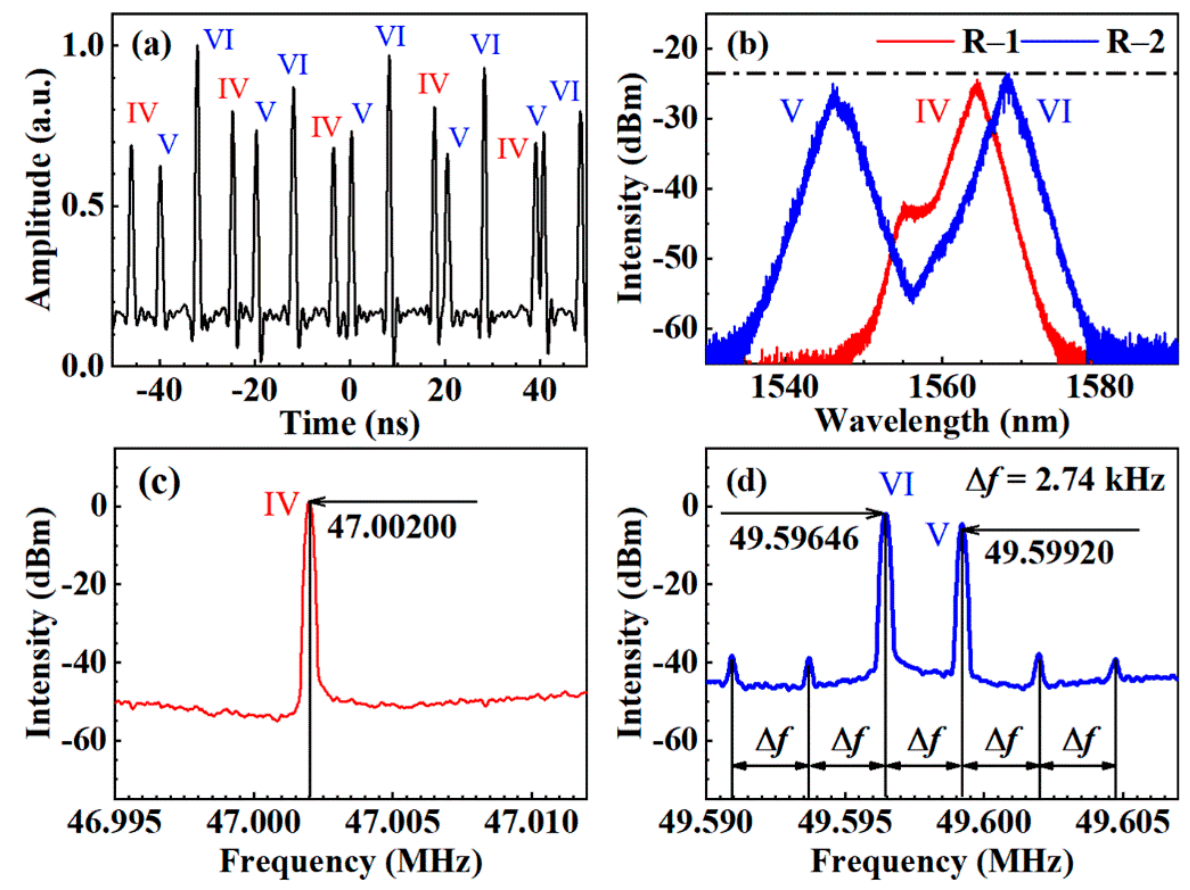

Figure 5. The output characteristics of the generated tri-comb with single-comb and dual-comb outputs from sub-ring 1 and sub-ring 2, respectively. (a) Pulse trains, (b) optical spectrum, (c) RF spectrum of sub-ring 1 and (d) RF spectrum of sub-ring 2. IV designates the pulse train from sub-ring 1, whereas V and VI designate the pulse trains from sub-ring 2. R-1, sub-ring 1. R-2, sub-ring 2.

\section{Conclusions}

In summary, a multidimensional multiplexing mode-locked laser based on a dual-ring integrative structure is proposed for tri-comb generation in this paper. To our knowledge, this is the first attempt to introduce sub-ring multiplexing into a multidimensional multiplexing mode-locked laser for simultaneous multi-comb generation. Combining sub-ring multiplexing and wavelength multiplexing, the proposed dual-ring integrated laser can generate a tri-comb through two modes. In the first mode, sub-ring 1 produced a dual-comb of $1569.1 \mathrm{~nm}$ and $1556.1 \mathrm{~nm}$ center wavelengths. The corresponding bandwidths of the optical spectrum were $1.4 \mathrm{~nm}$ and $3.3 \mathrm{~nm}$, respectively. The relative intensity of pulse trains and optical spectrum peaks were used to identify the generated combs. Based on the definition of dispersion, a rough analysis of the relationship between wavelength and equivalent refractive index was conducted to distinguish the repetition rates of $47.00198 \mathrm{MHz}$ and $47.00436 \mathrm{MHz}$ for the dual-comb above. Furthermore, the small peaks in the RF spectrum of the dual-comb were confirmed to be generated by extra interference among the RF signals. The single comb from sub-ring 2 showed a $1557.3-\mathrm{nm}$ center wavelength, 4.0-nm optical spectrum bandwidth and a 49.59724-MHz repetition rate. The minimum and maximum repetition rate differences of the generated tri-combs were $2.38 \mathrm{kHz}$ and $2.59526 \mathrm{MHz}$, respectively. In comparison, the other tri-comb generation mode was realized by sub-ring 2 producing a dual-comb. In this case, the minimum and maximum repetition rate differences of the generated tri-comb were able to be changed to $2.74 \mathrm{kHz}$ and $2.59720 \mathrm{MHz}$. This was achieved by switching the operation mode of the same dual-ring integrated mode-locked laser only. We believe the proposed new dimension of sub-ring multiplexing and the dual-ring integrated mode-locked laser could meet the needs of multi-comb generation with large-range repetition rate differences. Moreover, the repetition rate differences of generated combs can be freely adjusted by changing the resonance length of sub-rings with the proposed new dimension of sub-ring multiplexing. The obtained results indicate that our method can offer a powerful scheme for future multi-comb generation and its application in multi-heterodyne detection-based laser ranging and spectroscopy. 
Author Contributions: Conceptualization, R.Y.; methodology, R.Y. and H.S.; validation, H.S., H.L. and J.W.; formal analysis, R.Y. and H.S.; investigation, J.X.; resources, H.S. and H.L.; data curation, H.S. and H.L.; writing-original draft preparation, R.Y. and H.S.; writing-review and editing, H.Y. and H.F.; visualization, J.W.; supervision, J.T.; project administration, P.H.; funding acquisition, R.Y. and P.H. All authors have read and agreed to the published version of the manuscript.

Funding: This work was supported by the National Major Science and Technology Projects of China (2017ZX02101006-005), National Natural Science Foundation of China (NSFC) (51605120, 51675138, 61675058), China Postdoctoral Science Foundation (2018T110290), and the Fundamental Research Funds for the Central Universities (HIT. NSRIF. 2020103).

Conflicts of Interest: The authors declare no conflict of interest.

\section{References}

1. Coddington, I.; Swann, W.C.; Nenadovic, L.; Newbury, N.R. Rapid and precise absolute distance measurements at long range. Nat. Photonics 2009, 3, 351-356. [CrossRef]

2. Yang, R.; Pollinger, F.; Meiners-Hagen, K.; Tan, J.; Bosse, H. Heterodyne multi-wavelength absolute interferometry based on a cavity-enhanced electro-optic frequency comb pair. Opt. Lett. 2014, 39, 5834-5837. [CrossRef]

3. Wu, H.; Zhao, T.; Wang, Z.; Zhang, K.; Xue, B.; Li, J.; He, M.; Qu, X. Long distance measurement up to $1.2 \mathrm{~km}$ by electro-optic dual-comb interferometry. Appl. Phys. Lett. 2017, 111, 251901. [CrossRef]

4. Zhu, Z.; Wu, G. Dual-comb ranging. Engineering 2018, 4, 772-778. [CrossRef]

5. Xue, B.; Zhang, H.; Zhao, T.; Jing, H. A traceable high-accuracy velocity measurement by electro-optic dual-Comb Interferometry. Appl. Sci. 2019, 9, 4118. [CrossRef]

6. Keilmann, F.; Gohle, C.; Holzwarth, R. Time-domain mid-infrared frequency-comb spectrometer. Opt. Lett. 2004, 29, 1542-1544. [CrossRef]

7. Ideguchi, T.; Poisson, A.; Guelachvili, G.; Picqué, N.; Hänsch, T.W. Adaptive real-time dual-comb spectroscopy. Nat. Commun. 2014, 5, 3375. [CrossRef] [PubMed]

8. Coddington, I.; Newbury, N.R.; Swann, W.C. Dual-comb spectroscopy. Optica 2016, 3, 414-426. [CrossRef]

9. Zhao, X.; Qu, X.; Zhang, F.; Zhao, Y.; Tang, G. Absolute distance measurement by multi-heterodyne interferometry using an electro-optic triple comb. Opt. Lett. 2018, 43, 807-810. [CrossRef] [PubMed]

10. Liao, R.; Tian, H.; Liu, W.; Li, R.; Song, Y.; Hu, M. Dual-comb generation from a single laser source: Principles and spectroscopic applications towards mid-IR-A review. J. Phys. Photonics 2020, 2, 042006. [CrossRef]

11. Kieu, K.; Mansuripur, M. All-fiber bidirectional passively mode-locked ring laser. Opt. Lett. 2008, 33, 64-66. [CrossRef] [PubMed]

12. Ouyang, C.; Shum, P.; Wu, K.; Wong, J.H.; Lam, H.Q.; Aditya, S. Bidirectional passively mode-locked soliton fiber laser with a four-port circulator. Opt. Lett. 2011, 36, 2089-2091. [CrossRef] [PubMed]

13. Zeng, C.; Liu, X.; Yun, L. Bidirectional fiber soliton laser mode-locked by single-wall carbon nanotubes. Opt. Express 2013, 21, 18937-18942. [CrossRef] [PubMed]

14. Yao, X. Generation of bidirectional stretched pulses in a nanotube-mode-locked fiber laser. Appl. Opt. 2014, 53, 27-31. [CrossRef] [PubMed]

15. Zhao, X.; Zheng, Z.; Liu, Y.; Hu, G.; Liu, J. Dual-wavelength, bidirectional single-wall carbon nanotube mode-locked fiber laser. IEEE Photonics Technol. Lett. 2014, 26, 1722-1725. [CrossRef]

16. Liu, H.H.; Chow, K.K. Operation-switchable bidirectional pulsed fiber laser incorporating carbon-nanotube-based saturable absorber. IEEE J. Sel. Top. Quantum Electron. 2014, 20, 278-282. [CrossRef]

17. Krylov, A.A.; Chernykh, D.S.; Grebenyukov, V.V.; Arutyunyan, N.R.; Pozharov, A.S.; Obraztsova, E.D. Generation regimes of bidirectional hybridly mode-locked ultrashort pulse erbium-doped all-fiber ring laser with a distributed polarizer. Appl. Opt. 2016, 55, 4201-4209. [CrossRef]

18. Liu, B.; Luo, Y.; Xiang, Y.; Xiao, X.; Sun, Q.; Liu, D.; Shum, P.P. Multiplexed ultrafast fiber laser emitting multi-state solitons. Opt. Express 2018, 26, 27461-27471. [CrossRef]

19. Akosman, A.E.; Sander, M.Y. Dual comb generation from a mode-locked fiber laser with orthogonally polarized interlaced pulses. Opt. Express 2017, 25, 18592-18602. [CrossRef]

20. Zhao, X.; Li, T.; Liu, Y.; Li, Q.; Zheng, Z. Polarization-multiplexed, dual-comb all-fiber mode-locked laser. Photonics Res. 2018, 6, 853-857. [CrossRef] 
21. Nakajima, Y.; Hata, Y.; Minoshima, K. All-polarization-maintaining, polarization-multiplexed, dual-comb fiber laser with a nonlinear amplifying loop mirror. Opt. Express 2019, 27, 14648-14656. [CrossRef] [PubMed]

22. Zhao, X.; Zheng, Z.; Liu, L.; Liu, Y.; Jiang, Y.; Yang, X.; Zhu, J. Switchable, dual-wavelength passively mode-locked ultrafast fiber laser based on a single-wall carbon nanotube mode-locker and intracavity loss tuning. Opt. Express 2011, 19, 1168-1173. [CrossRef]

23. Zhao, X.; Hu, G.; Zhao, B.; Li, C.; Pan, Y.; Liu, Y.; Yasui, T.; Zheng, Z. Picometer-resolution dual-comb spectroscopy with a free-running fiber laser. Opt. Express 2016, 24, 21833-21845. [CrossRef] [PubMed]

24. Liao, R.; Song, Y.; Liu, W.; Shi, H.; Chai, L.; Hu, M. Dual-comb spectroscopy with a single free-running thulium-doped fiber laser. Opt. Express 2018, 26, 11046-11054. [CrossRef] [PubMed]

25. Li, R.; Shi, H.; Tian, H.; Li, Y.; Liu, B.; Song, Y.; Hui, M. All-polarization-maintaining dual-wavelength mode-locked fiber laser based on Sagnac loop filter. Opt. Express 2018, 26, 28302-28311. [CrossRef]

26. Cui, Y.; Liu, X. Graphene and nanotube mode-locked fiber laser emitting dissipative and conventional solitons. Opt. Express 2013, 21, 18969-18974. [CrossRef]

27. Yun, L.; Liu, X.; Han, D. Observation of vector- and scalar-pulse in a nanotube-mode-locked fiber laser. Opt. Express 2014, 22, 5442-5447. [CrossRef]

28. Li, T.; Zhao, X.; Chen, J.; Li, Q.; Xie, S.; Zheng, Z. Tri-Comb and Quad-Comb Generation Based on a Multi-Dimensional Multiplexed Mode-Locked Laser. J. Light. Technol. 2019, 37, 5178-5184. [CrossRef]

29. Yang, R.; Sun, H.; Hu, P.; Yang, H.; Fu, H.; Fan, Z.; Tan, J. Experimental exploration of mode-locking evolution mechanism in dual-ring fiber laser. Optik 2020, 208, 163899. [CrossRef]

30. Available online: https://www.corning.com/media/worldwide/coc/documents/Fiber/PI-1463-AEN.pdf (accessed on 12 October 2020).

31. Available online: https://www.thorlabschina.cn/newgrouppage9.cfm?objectgroup_id=739 (accessed on 23 October 2020).

32. Available online: https://refractiveindex.info/?shelf=glass\&book=SCHOTT-SF\&page=N-SF1 (accessed on 23 October 2020).

33. Available online: https://refractiveindex.info/?shelf=other\&book=air\&page=Ciddor (accessed on 23 October 2020).

34. Weiner, A.M. Dispersion and dispersion compensation. In Ultrafast Optics; Boreman, G., Ed.; John Wiley \& Sons, Inc. Publication: Hoboken, NJ, USA, 2009; pp. 147-151.

Publisher's Note: MDPI stays neutral with regard to jurisdictional claims in published maps and institutional affiliations.

(C) 2020 by the authors. Licensee MDPI, Basel, Switzerland. This article is an open access article distributed under the terms and conditions of the Creative Commons Attribution (CC BY) license (http://creativecommons.org/licenses/by/4.0/). 